\title{
Posterior dislocation of an intraocular lens implant and its removal
}

\author{
A. M. CHOWDHURY AND J. F. BRAS
}

SUMMARY The cause of dislocation of an intraocular lens is a direct blow or jerk, and dislocation may complicate both iris-supported and capsule-supported lenses. In capsule-supported lenses the implant dislocates towards the anterior chamber but in iris-supported lenses the dislocation is posterior. A technique for removal of a posterity dislocated lens is described.

In 200 cases of successful intraocular implants in the last five years three cases of dislocation of the lens have occurred, an incidence of $1.5 \%$. One was capsule-supported and the other two were irissupported.

The prime cause of dislocation of these lenses is a direct blow or jerk in association with a lax iris sphincter. In one case a jerk due to physical exercise caused an anterior dislocation of a capsule-supported implant lens. The other two cases involved irissupported implants, and the lenses dislocated posteriorly towards the vitreous cavity. In one the intact vitreous face held the implant against the iris; in the other the vitreous face was broken and the lens sank to the bottom of the vitreous chamber.

\section{Case report}

A male 51 years old developed a right monocular cataract over a two-year period. There was no history of injury or any obvious cause. In September 1975, after intracapsular extraction with peripheral iridectomy, a Binkhorst iris clip type of implant was used. Postoperative progress was satisfactory, and his visual acuity improved to $6 / 6$ in that eye. Unfortunately in March 1976 his hand slipped while pulling and hit his right eye with considerable force. This caused the implanted lens to dislocate posteriorly into the vitreous cavity. The vitreous face was broken and the lens sank into the fluid vitreous. The position of the lens in the vitreous cavity altered with every movement of the head and of the globe and with every change of position of the body in space.

\section{Removal technique}

The pupil was fully dilated, and retrobulbar anaesthesia was induced. Traction sutures were employed on the lids along with a superior rectus bridle suture. The patient was then turned over and made

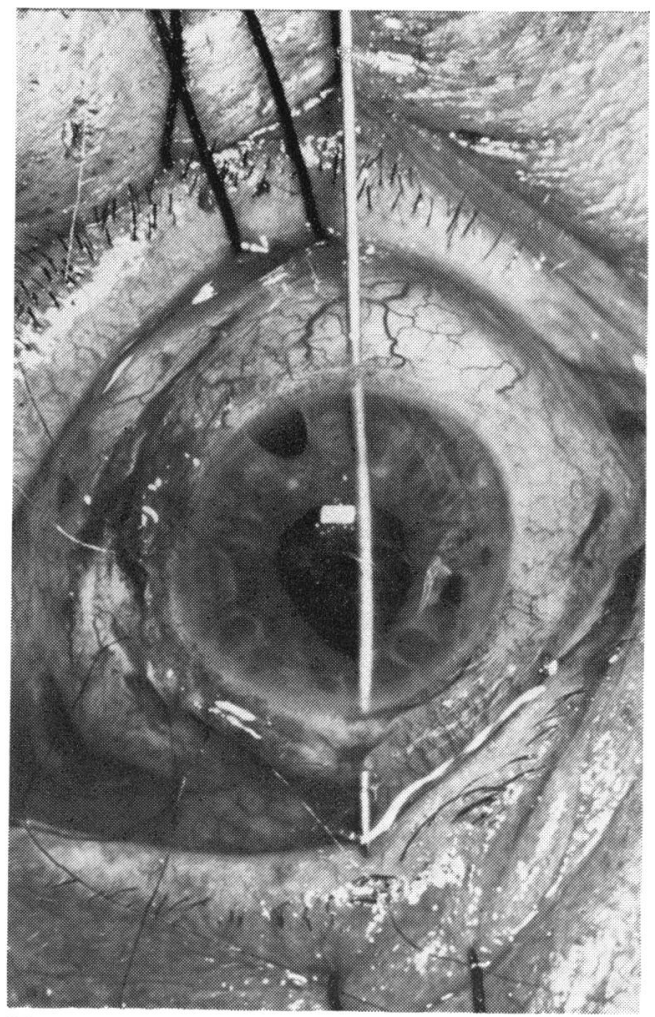

Fig. 1 The lens anchored by the needle 
to lie flat in the prone position with his head overhanging the top of the table. This procedure brought the dislocated pseudophakia downwards due to gravity, and a set of loops protruded into the anterior chamber through the dilated pupil.

To anchor the lens in that position an approach had to be made from below, and a needle (made from an ordinary retrobulbar needle) was passed through the limbus at the 1 o'clock position into the anterior chamber and through to the limbus at 7 o'clock. This was the most convenient position in this particular case, enabling the needle to pass through the loops of the implant and anchor it firmly (Fig. 1).

After the lens was anchored the patient was turned into the supine position. Sterile drapes were positioned and an ab-externo approach was made at the lateral limbus from 8 o'clock to 12 o'clock under a limbus-based conjunctival flap. A pair of curved, toothed iris forceps was introduced into the anterior chamber and the loops of the implant were grasped. The fixing needle was then withdrawn and the implant extracted slowly.

To close the wound the cornea was sutured with virgin silk and the conjunctiva with fine catgut. Postoperative progress was satisfactory, and the patient was discharged home within a week of the operation. Vision later improved to $6 / 12$ Snellen and N6 with a suitable reading addition. The patient has subsequently been fitted with a contact lens. 